Aksaray University
Journal of Science and Engineering
e-ISSN: 2587-1277
http://dergipark.gov.tr/asujse
http://asujse.aksaray.edu.tr

Research Article

\title{
On a Subspace Training Method for Dispersive DS-CDMA Channels
}

\author{
Ahmet Rizaner, Ali Hakan Ulusoy* \\ Department of Information Technology, Eastern Mediterranean University, Gazimagusa, North Cyprus, via \\ Mersin 10 Turkey \\ -Received Date: 18 May $2017 \quad$-Revised Date: 20 Jul $2017 \quad$ •Accepted Date: 16 Aug $2017 \quad$-Published Online:15 Sep 2017
}

\begin{abstract}
A subspace training method has been proposed for dispersive direct-sequence code division multiple access (DS-CDMA) channels. Its performance was compared to a matched filter receiver. We think that it is not quite fair to compare a minimum mean square error (MMSE) based receiver to a matched filter receiver. For this reason, we further investigate the method demonstrating that its performance significantly falls short of the true MMSE receiver and that it suffers from error floor. We also illustrate that any attempt to fill the gap significantly increases the system complexity and drops the efficiency of the transmission, which were originally mentioned as the advantages of the proposed method.
\end{abstract}

\section{Keywords}

CDMA, Multipath Channel, Channel Estimation

\section{INTRODUCTION}

High-rate code division multiple access (CDMA) systems suffer from two interferences; namely, multiple access interference (MAI) and inter-symbol interference (ISI) [1-3]. The MAI is due to the inherent non-orthogonality in CDMA systems, and the ISI is due to the multipath nature of the wireless channels. The suppression of both MAI and ISI needs joint multiuser detection and equalization $[2,3]$. This can be accomplished either by training using known

${ }^{*}$ Corresponding Author: alihakan.ulusoy@emu.edu.tr 
symbols or by operating in the blind mode. A joint blind equalization and multiuser detection based on a subspace approach has been presented in [4]. It is a two-stage block-by-block processing method. First, the effective signature waveform (convolution of the signature waveform and the channel impulse response) is estimated. Second, a linear detector (zero forcing (ZF) or minimum mean squared error (MMSE) detector) is formed. A training sequence based method that combines the two into a single step has been proposed [1]. The claim was that the resulting system is more computationally efficient and performs very well even with a few numbers of training symbols. However, the authors compared their system at a particular setup to the matched filter receiver only to reach that conclusion. We believe that it is not quite fair to compare an MMSE based receiver to a matched filter receiver. For this reason, we further investigate the method and show that the method suffers from error floor and performs substantially worse than the true MMSE detector, particularly at high signal to noise ratios (SNRs). Although it is possible to reduce the gap with the length of the analysis data block and the number of training symbols in the block, the former increases the system complexity and delay, and the latter reduces the transmission efficiency. We provide several simulation results to shed light on the behaviour of the system.

\section{SYSTEM MODEL}

In a CDMA system, several users transmit simultaneously over a common channel. The received baseband signal with a single receiver from $K$ users can be represented as the superposition of the data signals of the active users with additive white Gaussian noise, given by

$$
r(t)=\sum_{k=1}^{K} A_{k} \sum_{i=-\infty}^{\infty} b_{k}(i) \sum_{j=1}^{N} c_{k}(j) g_{k}\left(t-i T-j T_{c}\right)+n(t)
$$

where $n(t)$ is zero mean complex white Gaussian noise with a power spectral density $\sigma^{2}, A_{k}$ is the transmitted amplitude of the $k$ th user, $b_{k}(n)\left\{b_{k}(n) \in \pm 1\right\}$ are the information-bearing symbols, $\left\{c_{k}(j) \in \pm 1\right\}, j=1, \ldots, N$, is the pre-assigned spreading code of the $k$ th user, $N$ is the code length, $T$ is the symbol duration, $T_{c}$ is the chip duration and $g_{k}(t)$ is the impulse response of the channel. The dispersive CDMA channel is cast into the multiple-input multiple-output (MIMO) signal model framework reported in [1]. It is assumed that $g_{k}(t)$ has finite support of 
length $L T$. By stacking $m$ successive received samples, the received signal of the $l$ th symbol interval can be expressed in matrix form [1],

$$
\mathbf{r}(l)=\mathbf{H}(l) * \mathbf{b}(l)+\mathbf{n}(l)
$$

where * denotes convolution, $\mathbf{b}(l), \mathbf{n}(l)$ and $\mathbf{H}(l)$ are the data vector, noise vector and channel coefficients matrix of the $l$ th symbol interval defined in [1] respectively.

\section{SUBSPACE TRAINING METHOD}

By performing an eigen-decomposition of the autocorrelation matrix of the received signal $\mathbf{r}(l)$, we get

$$
\mathbf{R}=E\left\{\mathbf{r}(l) \mathbf{r}(l)^{H}\right\}=\mathbf{U} \boldsymbol{\Lambda} \mathbf{U}^{H}=\left[\begin{array}{ll}
\mathbf{U}_{\mathbf{s}} & \mathbf{U}_{\mathbf{n}}
\end{array}\right]\left[\begin{array}{ll}
\boldsymbol{\Lambda}_{\mathbf{s}} & \\
& \boldsymbol{\Lambda}_{\mathbf{n}}
\end{array}\right]\left[\begin{array}{l}
\mathbf{U}_{\mathbf{s}}^{\mathbf{H}} \\
\mathbf{U}_{\mathbf{n}}^{\mathbf{H}}
\end{array}\right]
$$

where $\mathbf{U}=\left[\mathbf{U}_{\mathrm{s}} \mathbf{U}_{\mathrm{n}}\right], \Lambda=\operatorname{diag}\left(\boldsymbol{\Lambda}_{\mathrm{s}}, \Lambda_{\mathrm{n}}\right)$, and $\Lambda_{\mathrm{s}}=\operatorname{diag}\left(\lambda_{1}, \lambda_{2}, \ldots, \lambda_{d}\right), d=K(m+L-1)$ contains the $d$ largest eigenvalues of $\mathbf{R}$ in descending order and $\mathbf{U}_{\mathrm{s}}=\left[\begin{array}{llll}\mu_{1} & \mu_{2} & \ldots & \mu_{d}\end{array}\right]$ contains the corresponding eigenvectors. Here, we have used the assumption that each user's information symbols are independent among themselves and are independent of the noise. The linear MMSE receiver of the $k$ th user, which minimizes the mean squared error (MSE), is given by [1]:

$$
\mathbf{z}_{k}=\mathbf{U}_{s}\left(\mathbf{U}_{s}^{H} \boldsymbol{\Gamma} \boldsymbol{\Gamma}^{H} \mathbf{U}_{s}\right)^{-1} \mathbf{U}_{s}^{H} \boldsymbol{\Gamma} \mathbf{b}_{k}
$$

where $\mathbf{b}_{k}=\left[\begin{array}{lllll}b_{k}(1) & b_{k}(2) & \ldots & b_{k}(P)\end{array}\right]^{T}$ is the vector of the training symbols of the $k$ th user, $\Gamma=\left[\begin{array}{llll}\mathbf{r}(1) & \mathbf{r}(2) & \ldots & \mathbf{r}(P)\end{array}\right]$ is the matrix of $P$ corresponding received data vectors and $P$ is the number of training symbols in each frame. The $i$ th bit detected of the $k$ th user can be estimated by $\hat{b}_{k}(i)=\operatorname{sgn}\left(\Re\left[\mathbf{z}_{k}^{H} \mathbf{r}(i)\right]\right)$.

\section{RESULTS AND DISCUSSIONS}

We provide some simulation examples to demonstrate the system performance with respect to the block size, $M$ (number of symbols used to estimate the MMSE detector), and the number of training symbols in the block, $P$. We compare the performance to that of the true MMSE detector [5], which assumes perfect knowledge of signature waveforms, gains and the channel, under the same experimental conditions. The experimental set up is very similar to that 
presented in [1]. We consider a five-user synchronous CDMA system. The desired and interfering users employed Gold sequences of length $N=31$ as spreading codes. The delay spread of the dispersive channel is one symbol interval and channels are assumed to be slowly varying. The smoothing factor is $m=2$ [1]. The preamble bits and data bits were randomly generated for each user and perfect power control (i.e. all users have equal powers, $A_{k}=1$ ) was assumed.

In Fig. 1, the bit error rate (BER) with respect to $M$ and $P$ is illustrated. The SNR was set to 10 $\mathrm{dB}$. The true MMSE detector performance is also included. It is clear that we need large values for both $M$ and $P$ to approach the performance of the true MMSE detector. Fig. 2 depicts the near-far resistance of the proposed method for several values of $M$ and $P$ at $10 \mathrm{~dB}$ SNR. The near-far ratio (the ratio of the average received power of each interferer to that of the desired user) changes from $-10 \mathrm{~dB}$ to $10 \mathrm{~dB}$. As evident from the simulation results, the algorithm is not near-far resistant and for strong MAI it fails to show satisfactory performance. Power control is necessary to improve the performance of all the users. In Fig. 3, we illustrate the system performance with respect to SNR for several values of $M$ and $P$. The error floor can be easily seen in the plot. It is obvious that we need large values of $M$ and $P$ to reduce the gap.

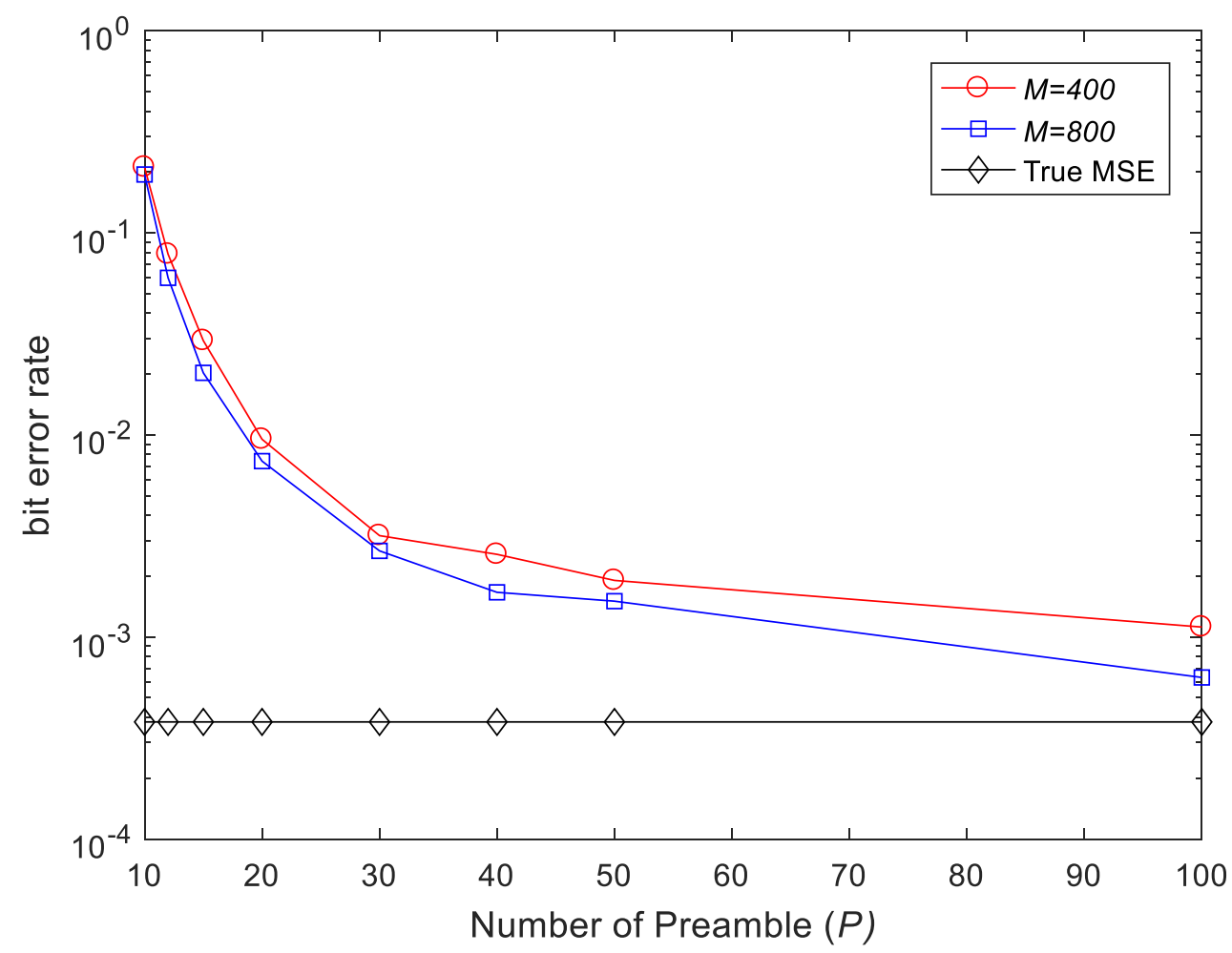

Figure 1. BER versus number of training symbols for user $1(\mathrm{SNR}=10 \mathrm{~dB})$ 


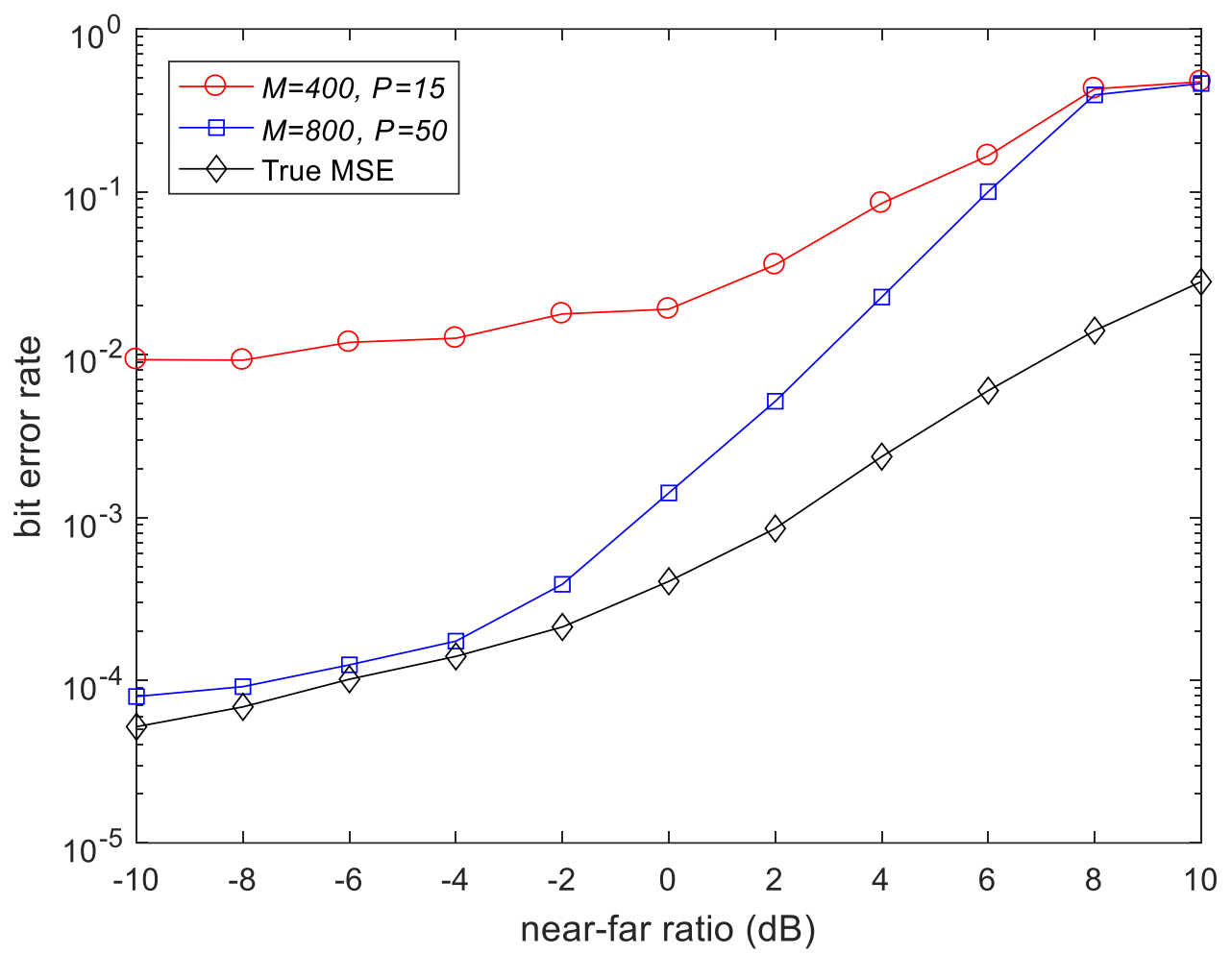

Figure 2. BER versus near-far-ratio for user $1(\mathrm{SNR}=10 \mathrm{~dB})$

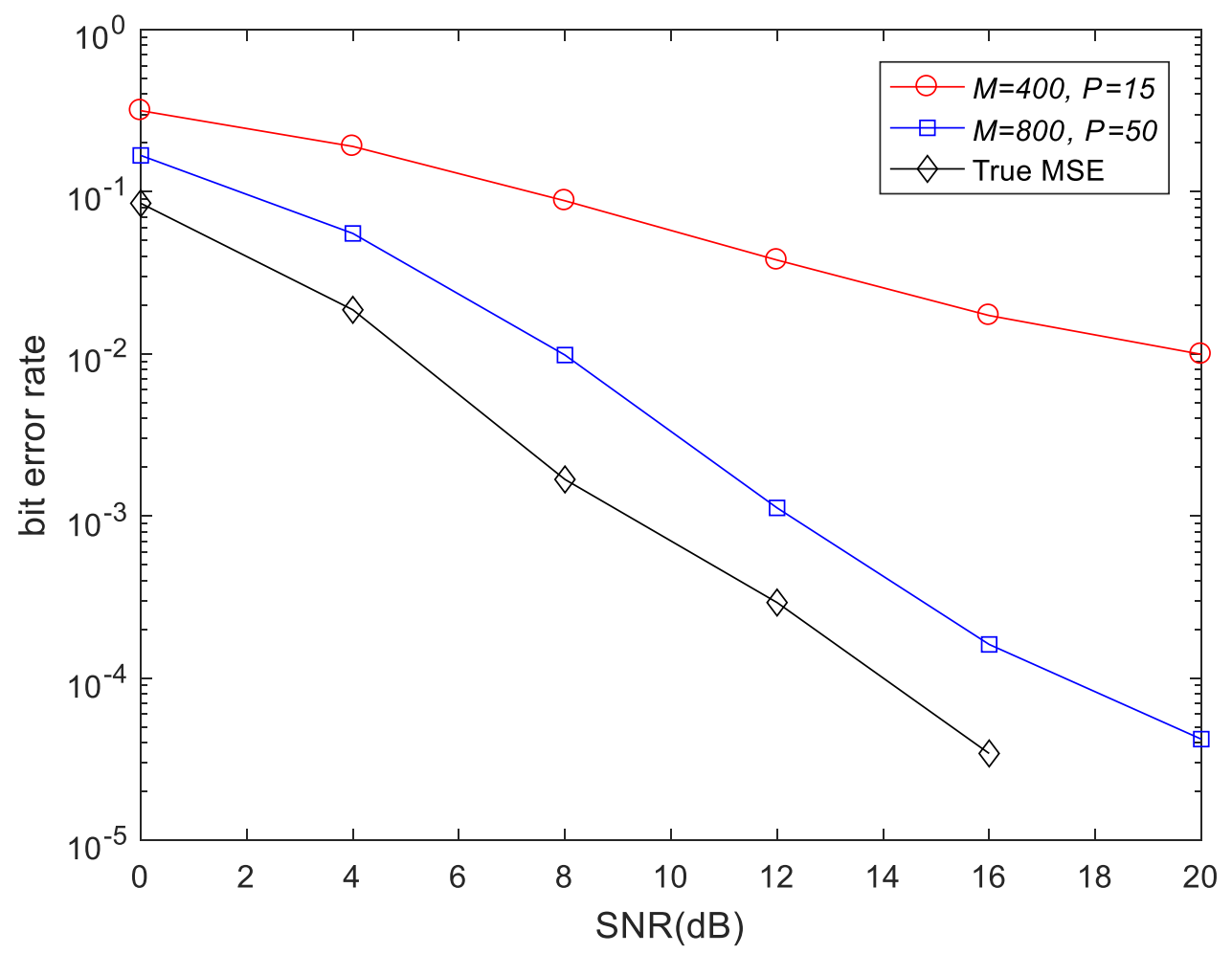

Figure 3. BER versus SNR for user 1 


\section{CONCLUSION}

The subspace training method proposed in [1] has been investigated. It has been demonstrated that its performance significantly falls short of the performance of the true MMSE receiver, particularly at high SNRs. In order to improve the performance, one requires a larger number of symbols in each frame and a longer sequence of training symbols for the estimation, which, in turn, they increase the system complexity and delay, and drop the efficiency of the transmission significantly.

\section{REFERENCES}

[1] Y. Zhang, G. Bi, N.P. Boon, Subspace training method for multiuser detection in dispersive CDMA channel. IEE Electronics Letters 36(15) (2000) 1315-1317.

[2] P. De, Computationally-efficient algorithms for multiuser detection in short code wideband CDMA TDD systems. Journal of Communications and Networks 18(1) (2016): 27-39.

[3] A. Agarwal, L.S. Purohit, L. Tharani, An approach to compare the developments of MAI cancellation in multiuser detection of DS-CDMA system, Soft Computing Techniques and Implementations (ICSCTI), 2015 International Conference on. IEEE, 2015.

[4] X. Wang, H.V. Poor, Blind equalization and multiuser detection in dispersive CDMA channels. IEEE Trans. on Communication 46(1) (1998) 91-103.

[5] M. Honig, K.K. Tsatsanis, Adaptive techniques for multiuser CDMA receivers. IEEE Signal Proc. Magazine (2000) 49-61. 